\title{
Controle Inteligente Aplicado a Sistemas Hidráulicos com Demanda Variável
}

\author{
Thommas K. S. Flores * Hugo A. M. Moreira* \\ Juan M. Villanueva* Heber P. Gomes* \\ * Universidade Federal da Paraíba, PB, (e-mail: \\ thommas.flores@cear.ufpb.br, HugoMarinho93@uol.com.br, \\ jmauricio@cear.ufpb.br, heberp@uol.com.br)
}

\begin{abstract}
Water supply systems are responsible for meeting the consumption demand of a population, but due to their habits this demand is variable. However, the energy expenditure in the pumping stage can reach up to $90 \%$, since in most cases the motor-pump assembly operates nominally, consequently raising service pressure at times of lower demand, wasting electrical energy and increasing leakage losses. In this panorama, this article presents the elaboration of a closed loop controller using fuzzy logic applied to the control of the rotation of a motor pump assembly present in a water pumping system with variable demand. This type of control arises as an alternative to increase the energy efficiency of the motor-pump assembly in front of a system without controller and controlled by manual registration, which the motorcycle pump in its nominal capacity. The results showed that the controller's performance showed a maximum increase of $9.16 \%$ in the motor pump set and a maximum reduction in the Specific Energy Consumption of $383 \%$ during the hours of minimum demand, in addition to reducing leakage losses, since the controller maintains constant pressure for 24 hours

Resumo: Os sistemas de abastecimento de água são responsáveis atender a demanda de consumo de uma população, porém devido aos seus hábitos esta demanda é variável. Entretanto, o gasto energético na etapa de bombeamento pode chegar até $90 \%$, pois na maioria dos casos o conjunto motobomba opera nominalmente, consequentemente elevando a pressão de serviço em horários de menor demanda, desperdiçando energia elétrica e aumentando as perdas por vazamento. Neste panorama, este artigo apresenta a elaboração de um controlador em malha fechada usando lógica fuzzy aplicado ao controle da rotação de um conjunto motobomba presente em um sistema de bombeamento de água com demanda variável. Este tipo de controle surge como alternativa para o aumento da eficiência energética do conjunto motobomba frente a sistema sem controlador e controlado por registro manual, cuja o conjunto motobomba em sua capacidade nominal. Os resultados apontaram que ação do controlador apresentou um aumento máximo do rendimento do conjunto motobomba de 9,16\% e redução máxima do Consumo Específico de Energia de 383\% durante o horário de mínima demanda, além de diminuir as perdas por vazamento, pois o controlador mantém a pressão constante durante as 24 horas.
\end{abstract}

Keywords: Fuzzy control; water supply systems; efficiency; variable demand; Specific Energy Consumption.

Palavras-chaves: Controle fuzzy; sistemas de abastecimento de água; eficiência; demanda variável; Consumo Específico de Energia.

\section{INTRODUÇÃO}

Os sistemas de abastecimento de água e de esgotamento sanitário são fundamentais manutenção e bem estar social. Para tanto, estes sistemas necessitam de uma grande quantidade de energia elétrica, sendo eles responsáveis por, aproximadamente, $3 \%$ da energia consumida no mundo, sendo $97 \%$ deste percentual utilizado na etapa de bombeamento (Gomes et all. (2012)).

A Agência Nacional de Energia Elétrica (ANEEL) estima que a indústria responsável por consumir $35,83 \%$ da energia distribuida em 2017 (ANEEL (2018)). Sabendo disso, estabeleceu que as empresas de distribuição de energia elétrica no Brasil implante um mecanismo chamadas de
Bandeiras Tarifárias, o qual estabelece uma variação no preço da energia elétrica a partir da expectativa de chuva (Lima et all. (2017)).

Dessa forma. as variações nas tarifas se deve ao fato de que $67 \%$ da energia produzida no território nacional é proveniente de hidrelétricas, logo em épocas onde há escasses de chuva, o custo da energia elétrica pode aumentar cerca de $12 \%$ em comparação a épocas chuvosas, este aumento é consequência do acionamento de fontes secundárias de alto custo para produção de energia elétrica, como por exemplo as termoelétricas (Lima et all. (2017)).

Neste contexto, a fim de amenizar o valor final do consumo de energia elétrica pago devido as variações tarifárias, 
torna-se necessário a elaboração de estratégias que visam melhorar a eficiência energética nos sitemas de bombeamento de água, isto é, diminuindo as perdas hidráulicas ao longo de toda rede de distribuição e adotando estratégiam e técnicas adequadas de operação e controle dos dispositivos utilizados no bombeamento.

Uma destas tecnicas apoia-se no escalonamento da operação das bombas com rotação fixa ao longo do dia, isto é, dependendo da demanda são acionadas ou desligadas determinados números de bombas, o resultado desta estratégia aumentou a eficiência energética em média $15 \%$ e no máximo em 25\%, quando comparado a operação sem escalonamento (Luna et all. (2019)).

Além disso, os sistemas de abastecimento de água apresentar caracteríticas de alta não linearidade e variantes no tempo, pois é dependente da demanda ao longo do dia, falhas, manutenção e diferentes pontos de operação dos diversos dispositivos que compõe o sistema. Portanto, técnicas de monitoramento, controle e operação são necessárias a fim de minimizar os custos, aumentar a eficiência e garantir as condições necessárias para atender o consumidor final em quantidade e qualidade (Babunski et all. (2018)).

Devido ao alto custo energético, métodos que utilizam inversores de frequência (Brogan et all. (2016)) unido a técnicas de controle em malha fechada (Redes Neurais Artificiais, Lógica fuzzy, Algoritmo Genético e Proporcional Integral e Derivativo) para controlar a velocidade do conjunto motobomba são amplamente utilizados para o aumento da eficiência energética (Page et all. (2017), Diniz et all. (2015) e Anjana et all. (2017)).

Ademais, é também observado o uso frequente de válvulas redutoras de pressão para o gerenciamento da pressão e vazão ao longo da rede de distribuição de água, porém estas introduzem perdas no sistema, consequentemente reduzindo a eficiência (Signoreti et all. (2016).

Neste panorama, este artigo apresenta a elaboração de um controlador fuzzy em malha fechada aplicado ao controle da rotação de um conjunto motobomba (CMB) presente em um sistema de bombeamento de água com demanda variável.

Este sistema de controlado surge como alternativa para o aumento da eficiência energética do sistema de bombeamento, os quais normalmente operando com a velocidade nominal do CMB durante todo o processo e/ou fazem o controle manual da pressão na saída do reservatório por meio de um registro manual.

Com a finalidade de avaliar a metodologia proposta foram realizados a análise hidroenergética por meio do cálculo do rendimento do conjunto motobomba e do Consumo Específico de Energia (CE), o qual quantifica a energia necessária por volume de água bombeada (Alegre et all. (2004)). Os resultados foram obtidos, a partir do estudo da dinâmica da demanda de água durante 24 h de operação de um sistema de abastecimento de água. Os ensaios experimentais foram realizados no sistema de bombeamento de água do Laboratório de Eficiência Energética e Hidráulica em Saneamento da Universidade Federal da Paraíba
(LENHS/UFPB), o qual é capaz de emular uma rede de distribuição de água que será usado neste trabalho.

\section{SISTEMAS DE ABASTECIMENTO DE ÁGUA}

Os sistemas de abastecimento de água são compostos, basicamente, por um ponto de captação da água bruta do meio ambiente, estação de tratamento e, por fim, distribuição por meio de ductos até os consumidores em quantidade e qualidade satisfatória para atender a demanda de consumo (Gomes et all. (2012)).

Na Fig. 1 está ilustrado um esquema hidráulico de um sistema de bombeamento, em que o conjunto motobomba é responsável por fornecer energia hidráulica, na forma de pressão e vazão, suficiente à água para vencer os desníveis geográficos, perda de carga e ainda chegar com pressão suficiente aos consumidores finais, a soma destas variáveis é denotado por altura manométrica total $(H)$ e usualmente utiliza-se a unidade de metros de coluna de água (mca) (Gomes et all. (2012)).

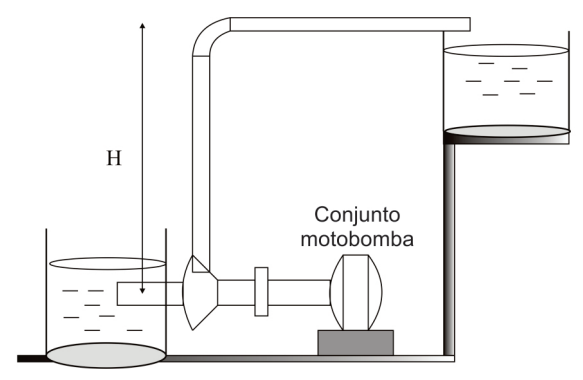

Figura 1. Esquema hidráulico de um sistema de bombeamento.

\section{PROJETO DO CONTROLADOR FUZZY}

Nesta seção será apresentada o projeto do controlador fuzzy para o controle da pressão de um sistemas de abastecimento de água, onde a malha de controle utilizada está ilustrada na Fig. 2, sendo os sinais de entrada do controlador o erro $\left(\left(e=H^{*}-H\right)\right)$, isto é, a diferença entre a pressão desejada $\left(H^{*}\right)$ e a pressão na saída do sistema $(H)$ e a variação do erro $(\Delta e=e(k)-e(k-1))$. Já o sinal de saída é o incremento da frequência $(\Delta f)$. A planta recebe como sinal de atuação a frequência de rotação do CMB $(f(k)=\Delta f+f(k-1))$ e tem como resposta a pressão na saída do sistema, a qual deseja-se controlar.

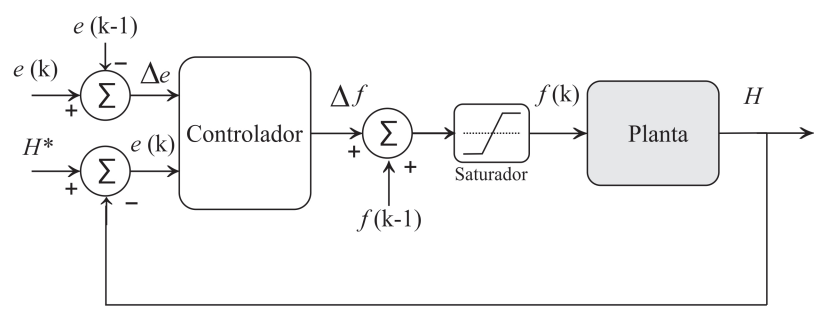

Figura 2. Sistema de controle do tipo MISO.

\subsection{Construção das funções de pertinência}

Com a finalidade de construir a estrutura do controlador fuzzy, inicialmente foi realizado a análise temporal da 
resposta da planta em malha a um degrau, e a partir disto foi definido um conjunto de funções de pertiência das variaveis de entrada e de saída do controlador. Posteriormente, foram estabelecidas as regras por meio do conhecimento do comportamento da planta e as ações de controle necessárias para o controle da pressão na saída do sistema.

Portanto, as variáveis linguísticas utilizadas para a variáveis de entrada são: Negativo Grande (NG), Negativo Médio (NM), Negativo Pequeno (NP), Zero (Z), Positivo Pequeno (PP), Positivo Médio (PM) e Positivo Grande (PG), conforme exposto de maneira gráfica na Fig. 3.
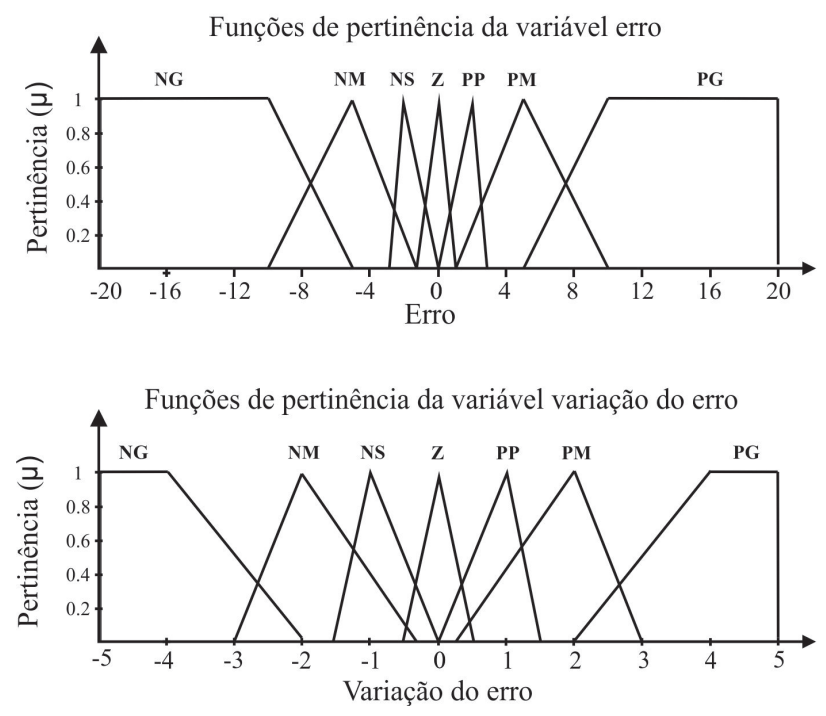

Figura 3. Funções de pertinência das variáveis de entrada.

Em contrapartida, por se tratar de uma malha de controle incremental, isto é, a saída do controlador fuzzy $(\Delta f)$ soma-se com a frequência de rotação anterior $(f(k-$ 1)), as funções de pertinência da variável de saída foram determinadas de modo empirico, sendo seu maior valor de incremento igual a $10 \mathrm{~Hz}$, conforme ilustrado na Fig. 3. Em que as variáveis linguisticas são: Decremento Pequeno (DP), Decremento Médio (DM), Decremento Grande (DG), Zero (Z), Incremento Grande (IG), Incremento Médio (IM) e Incremento Pequeno (IS).

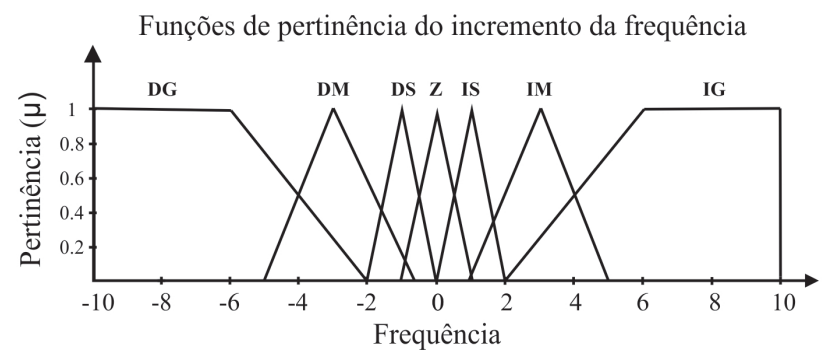

Figura 4. Funções de pertinência das variável de saída.

\subsection{Criação das regras}

A elaboração das regras teve como objetivo obter uma resposta de primeira ordem e com erro próximo de zero. Estas características são decorrentes do estudo da característica do sistema, pois a presença de um elevado sobressinal e um rápido aumento da pressão pode gerar um rompimento dos ductos, causar cavitação e prejudicar a motobomba devido a sobrecorrente sazonal durante a atuação do controle.

Portanto, é possível observar na Tab. 1 as 49 regras produzidas, com o proposito de estabilizar o sistema em regime permanente, além de apresentar uma resposta suave entre as regras de saída em função das regras de entrada.

Tabela 1. Regras do Controlador fuzzy.

\begin{tabular}{|c|c|c|c|c|c|c|c|c|}
\hline & \multicolumn{7}{|c|}{ Variação do erro } \\
\hline \multirow{4}{*}{ Erro } & & NB & NM & NS & Z & PS & PM & PB \\
\cline { 2 - 9 } & NB & DS & DS & DS & DM & DM & DB & DB \\
\cline { 2 - 9 } & NM & Z & DS & DM & DM & DM & DB & DB \\
\cline { 2 - 9 } & NS & Z & Z & DS & DS & DS & DS & DM \\
\cline { 2 - 9 } & Z & IS & Z & Z & Z & Z & Z & DS \\
\cline { 2 - 9 } & PS & IS & IS & IS & Z & IS & Z & Z \\
\cline { 2 - 9 } & PM & IB & IB & IM & IM & IM & IM & IS \\
\cline { 2 - 9 } & PB & IS & IB & IB & IB & IM & IM & IM \\
\hline
\end{tabular}

Por fim, durante o processo de inferência fuzzy, utilizou-se o método de Mamdani e o cálculo do centro de massa para a etapa de defuzzyficação, a dedução matemática destes métodos podem ser encontradas em Ross (2004).

\section{INDICADORES HIDROENERGÉTICOS}

Os indicadores de desempenho hidroenergéticos são métodos que permitem avaliar o comportamento das diversas características do sistema de bombeamento, auxiliando na identificação de falhas, aumentando a confiabilidade e aumentando o rendimento deste sistema (Alegre et all. (2004)).

Sendo assim, uma destes indicadores é o Consumo Específico de Energia (CE), o qual representa a quantidade de energia necessária para bombear um dado volume de água, isto é, repercutindo a eficiência do conjunto motobomba unida à eficiência do sistema hidráulico a jusante do dipositivo de bombeamento e é expresso por meio da equação (1) (Alegre et all. (2004) e Gomes et all. (2012)).

$$
C E=\frac{\text { Consumo de energia }}{\text { Volume bombeado }}
$$

Por outro lado, é possível quantificar a eficiência do sistema de distribuição de água por meio do cálculo do rendimento $(\eta)$ do conjunto motobomba por meio equação (2), a qual relaciona a potência hidráulica $\left(P_{h i d}\right)$, isto é, por meio da pressão $(H)$, vazão $(Q)$, peso específico do líquido $(\gamma)$ e a potência elétrica ativa $(P)$ (Gomes et all. (2012)).

$$
\eta=\frac{\gamma Q H}{P}=\frac{P_{h i d}}{P}
$$

A quantificação das características destes sistemas é fundamental para aprimoramento energético sistemas, principalmente em relação aos conjuntos motobombas, pois segundo Gomes et all. (2012) estas consomem até 90\% da energia elétrica de uma unidade de bombeamento.

Neste contexto, na Fig. 5 ilustra-se o comportamento da demanda de água ao longo do dia, em que o coeficiente $k$ é obtido pela razão entre o consumo do horário avaliado pelo consumo médio do dia. 


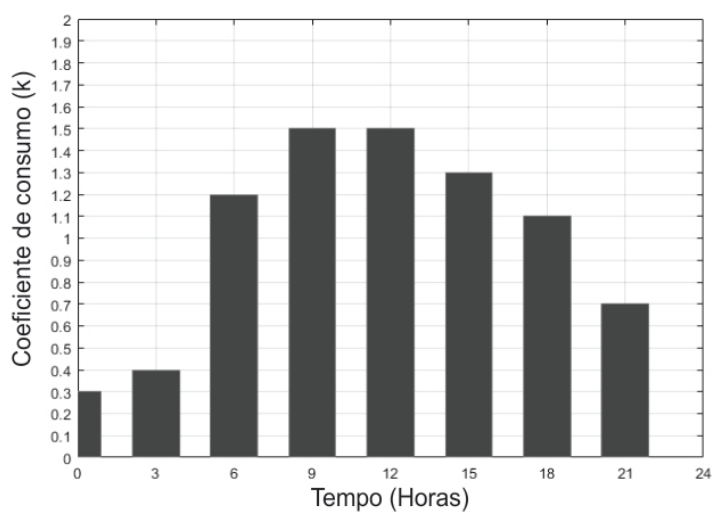

Figura 5. Hidrograma normalizado (Gomes et all. (2012)).

\section{SISTEMA EXPERIMENTAL}

Este trabalho foi desenvolvido em uma bancada experimental do Laboratório de Eficiência Energética e Hidráulica em Saneamento da Universidade Federal da Paraíba LENHS/UFPB. Tendo como objetivo emular um sistema de bombeamento de água totalmente instrumentalizado, permitindo a analise de diversos cenários de consumo, além de possibilitar o monitoramento dos parâmetros hidráulicos (pressão e vazão) e elétricos (potência) dos dispositivos presentes neste sistema. Na Fig. 6 se ilustra o diagrama da planta experimental, que contém um reservatório de armazenamento de água, sensores, atuadores, circuito de condicionamento e sistema de aquisição de dados.

Dessa forma, a água proveniente do reservatório é bombeada por uma bomba centrifuga (trifásica 220/380 V de $3 \mathrm{hp}$ ) através de canos e conexões em Polyvinyl chloride (PVC), a bomba fornece ao líquido energia em forma de pressão e vazão, que são mensuradas por meio de transdutores de pressão e vazão presentes neste sistema. A velocidade de rotação da bomba centrifuga é controlada por meio de um inversor de frequência. Além disso, na saída do sistema há uma válvula proporcional automatizada, a qual serve para emular a demanda de água ao regular a área de seção transversal por onde o fluido passa.

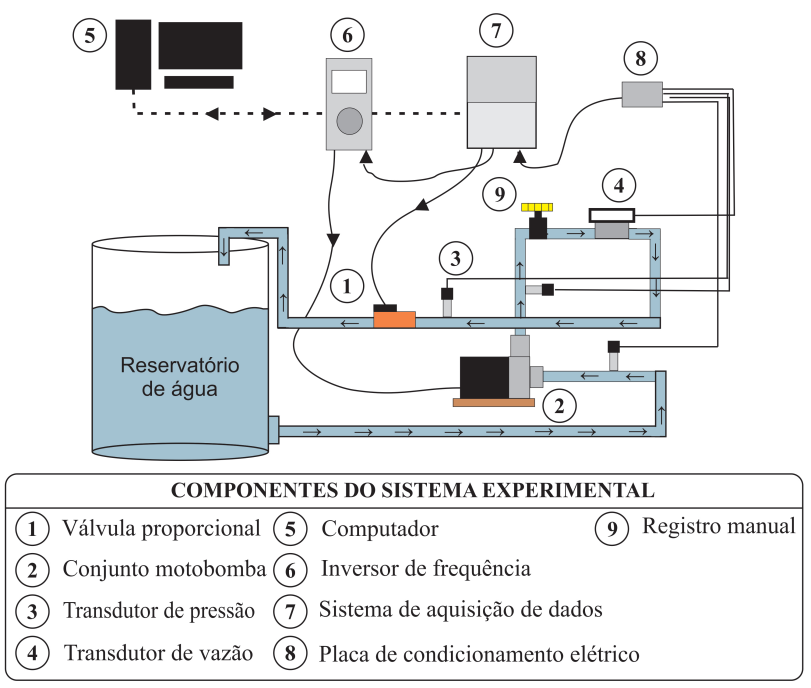

Figura 6. Esquema do sistema experimental.
Os sinais elétricos advindos dos sensores em forma de corrente são convertidos em tensão por meio de uma placa de condicionalmento elétrico, que em seguida são convertidos em um sinal digital e processados por um sistema de aquisição de dados (NI-USB 6009). Por fim, estes sinais são enviados até um computador (PC) para o registro das medições usando o supervisório do sistema. Os sinais que comandam o inversor de frequência e da válvula proporcional são geridos pelo sofware supervisório do mesmo PC e enviados aos dispositivos de atuação por meio da placa de aquisição de dados, ou seja, convertendo o valor digital do comando em um sinal elétrico analógico.

\section{RESUlTAdOS E DISCUSSÕES}

Nesta seção, serão apresentados os resultados obtidos durante os experimentos, em primeiro lugar será apresentado as características da resposta do sistema operando com o controlador de pressão proposto e em seguida o impacto hidroenergético na gestão da demanda de um sistema de bombeamento de água em três situações distintas de controle da vazão demandada, sendo:

- Conjunto motobomba operando com a velocidade nominal e na ausência de qualquer tipo de controle de vazão e/ou pressão;

- Conjunto motobomba operando com a velocidade nominal e a vazão do sistema controlado por meio de um registro manual;

- Conjunto motobomba acionado por um inversor de frequência, o qua está sujeito a um controle inteligente em malha fechada baseado em lógica fuzzy.

Sendo assim, modificação do ângulo da válvula proporcional (VRP) presente na saída da bancada experimental, permite emular a demanda variável de água ao longo do dia, isto é, quando a VRP está em $90^{\circ}$ significa que a passagem do fluido está totalmente obstruída e $0^{\circ}$ totalmente desobstruída. Então, adotou-se os ângulos de $30^{\circ}$ a $80^{\circ} \mathrm{com}$ mudança a cada $5^{\circ}$, este procedimento teve como objetivo identificar a vazão média experimental $\left(Q_{\text {med }}\right)$, resultando em $Q_{m e d}=0,7651 \mathrm{l} / \mathrm{s}$.

Em seguida, de posse da vazão média experimental $\left(Q_{m e d}\right)$ e sabendo o coeficiente de consumo $(k)$, proveniente do gráfico da Fig. 5, pode-se calcular a vazão experimental $\left(Q_{n}\right)$ por meio meio da equação $Q_{n}=k \cdot Q_{\text {med }}$, em que $n$ é a hora avaliada, que neste caso é a cada 3 horas do dia.

\subsection{Análise do controlador fuzzy}

Este experimento foi realizado com a motobomba partindo do repouso, isto é, com velocidade de rotação igual a zero e com o ângulo da válvula proporcional igual a $30^{\circ}$. O valor desejado da pressão foi de 10 mca (metros de coluna d'Água), em que é possível observar a resposta dinâmica do sistema ao degrau nas Fig. 7. Os resultados obtidos durante o transitório e o regime permanente estão na resumidos na Tab. 2.

\subsection{Analise Hidroenergética}

O resultado apresentado na Fig. 8 consiste no cálculo do rendimento do conjunto motobomba nas três situações 


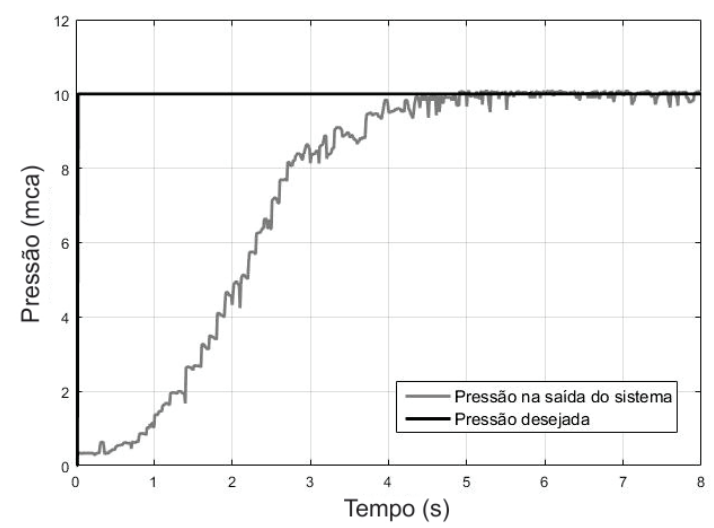

Figura 7. Esquema do sistema experimental.

Tabela 2. Características do sistema.

\begin{tabular}{|c|c|}
\hline Caracteristica & Resposta do sistema \\
\hline Tempo de subida & $1,76 \mathrm{~s}$ \\
\hline Tempo de assentamento & $4,35 \mathrm{~s}$ \\
\hline Sobressinal & - \\
\hline Erro em regime permanente & $0,79 \%$ \\
\hline
\end{tabular}

citadas no começo desta secção, para este cálculo usou-se a equação (2). Nesta figura pode-se obervar que durante os horários de baixa demanda o rendimento do sistema com controle fuzzy é superior frente aos outros modos de operação. Entretanto, este rendimento é inferior nos horários de máxima demanda. No primeiro caso, a diferença máxima que há entre os rendimentos se dá as 00:00 horas, sendo de $9,16 \%$. Já no segundo caso a diferença máxima é de $3,88 \%$.

O aumento rendimento durante a miníma demanda citado anteriormente, deve-se ao fato do controlador manter a pressão em 10 mca. Em contrapartida nos outros dois casos o CMB opera sempre em velocidade nominal, independente da demanda, aumentando a perdas por vazamento ao longo da rede de distribuição, principalmente nos horários de mínima demanda.

Por outro lado, quando o sistema opera com o CMB operando em velocidade nominal e a demanda é controlada por registro manual, há uma queda da pressão no usuário final, devido ao excesso de perda de carga causada pelo registro, consequentemente impactanto negativamento no rendimento global do sistema.

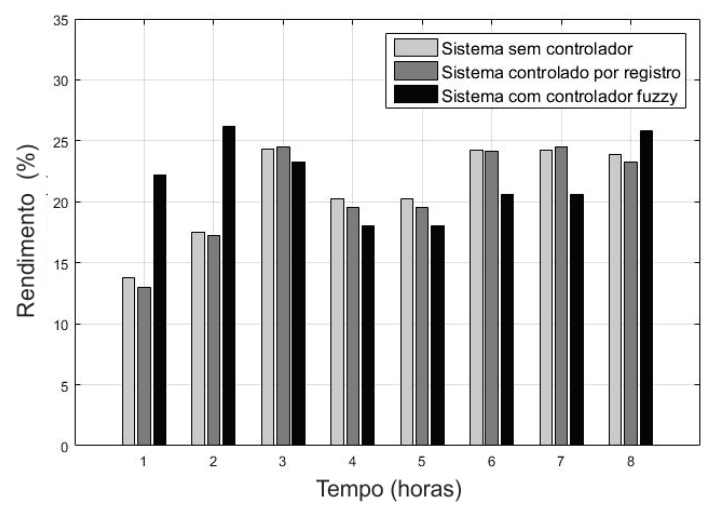

Figura 8. Rendimento do conjunto motobomba.
Na Fig. 9 ilustra-se a avaliação da potência elétrica ativa que o inversor de frequência fornece ao conjunto motobomba (CMB) para os três casos estudados. É notável que em qualquer período a potência consumida do sistema com controle fuzzy é menor ou igual, quando comparada com as outras duas metodologias adotadas neste trabalho. Este fato é atribuído a que quando usado controle fuzzy a velocidade de trabalho do CMB é variável e depende da demanda solicitada. Por outro lado, os outros dois métodos a velocidade de rotação do CBM sempre fixa e nominal, mesmo com menor demanda solicitada.

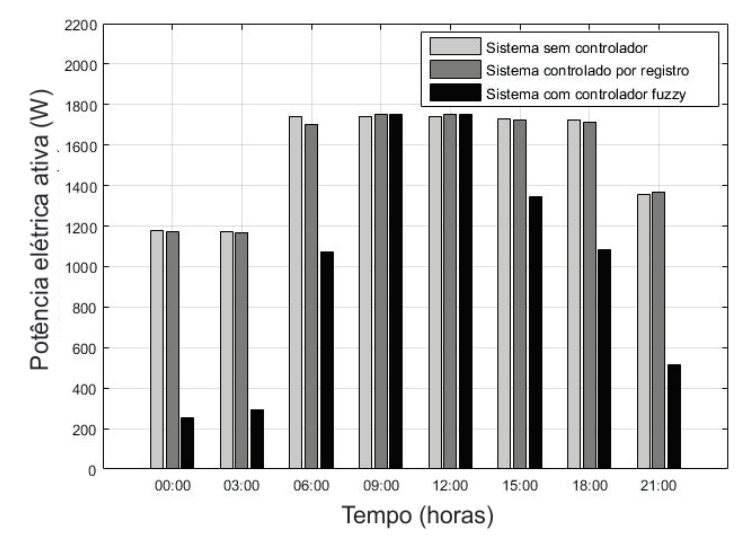

Figura 9. Potência elétrica ativa fornecida ao CMB.

Ademais, por meio da equação 1 foi realizado o cálculo do Consumo Específico de Energia dos 3 casos estudados, quanto menor o valor de $\mathrm{CE}$, maior será a eficiência do sistema de bombeamento.

Na Fig. 10 ilustra-se o resultado da avaliação do consumo específico de energia (CE), na qual se evidencia que a atuação do controlador fuzzy produz um CE menor ou igual quando comparado com as outras duas metodologias experimentadas. No período avaliado das 9:00 às 14:59, observasse a igualdade do CE nos três métodos adotados, isso porquê, em todos os casos, o sistema operam com velocidade nominal. Além disso, é possível constatar uma redução máxima de 383\% as 00:00, ao comparar o sistema operando com o controlador de pressão fuzzy e controlado manualmente através de um registro.

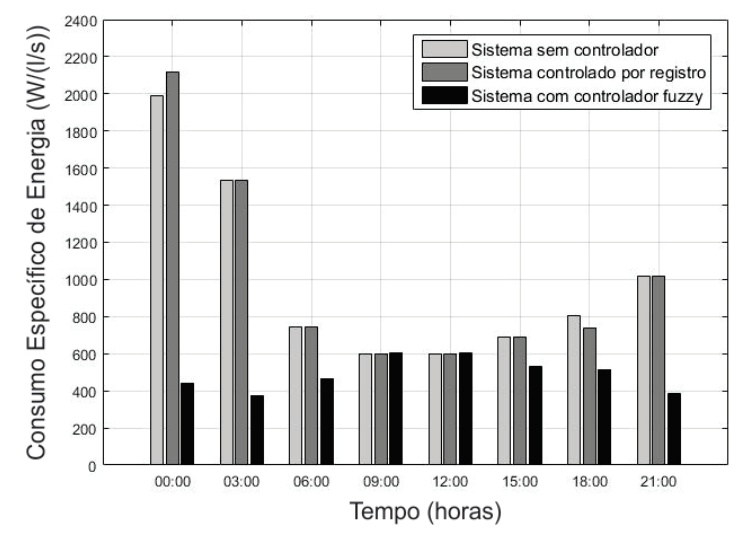

Figura 10. Consumo Específico de Energia.

Por fim, investigou-se o comportamento da pressão na saída do sistema ao longo das 24 h para cada um dos 3 
metódos de operação propostos neste artigo, cujo resultado é ilustrado na Fig. 11. Onde constatou que o sistema sem o controlador produz as maiores pressões durante os períodos de menores demandas, isto pode ocasionar rompimento nos ductos, aumentar os vazamentos e diminuir a eficiência.

Ademais, a demanda controlada por meio de um registro ocasiona diminuição na pressão fornecida para o usuário final, isto é, o registro controla a vazão demandada em custa de uma perda de carga excessiva, que segundo Gomes et all. (2012) o usuário deve receber uma pressão de no mínimo 10 mca em sua residência. Observando-se a Fig. 11 este valor de pressão só é atendido no período de 9:00 a 12:00, isto é, quando o sistema exige a máxima demanda, e quando o registro estará totalmente aberto.

Por outro lado, ao aplicar o controlador fuzzy no sistema, pode-se observar na Fig. 11 que a pressão mantém-se constante em $10 \mathrm{mca}$, atendendo o mínimo requisitado para atender o usuário final, por consequência reduz as perdas de água e aumenta a eficiência energética do sistema.

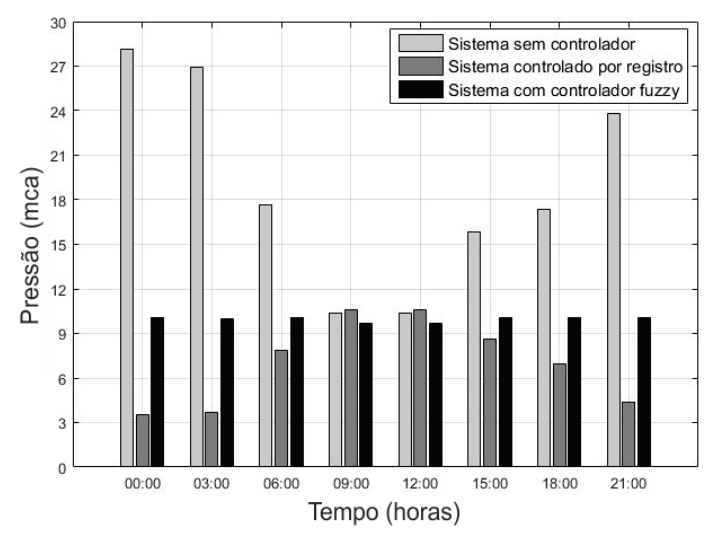

Figura 11. Pressão na saída do sistema.

\section{CONCLUSÃO}

Em vista dos resultados obtidos neste artigo, pode-se concluir que o controlador fuzzy apresentou comportamento adequado, isto é, não divergindo do valor desejado e atendeu os requisitos impostos pelo projetista. Além disso, foi observado que o desempenho do controlador fuzzy depende fortemente da base de regras, isto é, requer-se de um bom conhecimento da operação do sistema para a construção das regras.

Com relação a analise hidroenergética, é notório a melhoria na eficiência energética ao aplicar um controle em malha fechada unido a ação de um inversor de frequência para variação da velocidade de rotação do conjunto motobomba. Foi observado um aumento máximo de 9,16\% do rendimento e redução máxima de 1730 do Consumo Específico de Energia durante o horário de mínima demanda frente aos outros dois métodos apresentado neste trabalho. Assim também, o controle fuzzy possibilitou a constância na pressão de saída da rede hidráulica, consequentemente reduzindo as perdas por vazamento e rompimento dos ductos. Este fato, contribuiu para a eficácia do sistema de distribuição de água.

\section{AGRADECIMENTOS}

Os autores agradecem a Coordenação de Aperfeiçoamento de Pessoal de Nível Superior (CAPES), Laboratório de Eficiência Energética e Hidráulica em Saneamento (LENHS) e ao Grupo de Inteligência Computacional Aplicada (GICA) da Universidade Federal da Paraíba (UFPB) pelo suporte para a realização deste trabalho.

\section{REFERENCIAS}

Agência Nacional de Energia Elétrica (ANEEL). Anuário Estatístico de Energia Elétrica. Brasil, 2018.

H. P. Gomes, P. D. Carvalho. Manual de Sistema de Bombeamento-Eficiência Energética. João Pessoa: Editora da UFPB, 2012.

D.A. Lima, R.C. Perez and G. Clemente. A comprehensive analysis of the Demand Response Program proposed in Brazil based on the Tariff Flags mechanism. Electric Power Systems Research, volume 144, pages 1-12, 2017.

D. Babunski, E. Zaev, A.Tuneski and D. Bozovic. Optimization methods for water supply SCADA system. In 2018 \%th Mediterranean Conference on Embedded Computing (MECO), pages 1-4, June 2018.

T. Luna, J. Ribau, D. Figueiredo and R. Alves. Improving energy efficiency in water supply systems with pump scheduling optimization. Journal of Cleaner Production, 213, pages 342-356, 2019.

A. Brogan, V. Gopalakrishnan, K. Sturtevant, Z. Valigosky and K. Kissock. Improving Variable-Speed Pumping Control to Maximize Savings. ASHRAE Transactions, volume 122, n. 2, 2016.

P. R. Page, A. M. Abu-Mahfouz and M. L. Mothetha. Pressure management of water distribution systems via the remote real-time control of variable speed pumps. Journal of Water Resources Planning and Management, volume 143, n. 8, page 04017045, 2017.

A. M. F. Diniz, C. H. O. Fontes, C. A. Costa, A. M. S. Ferreira and P. I. D. Santos Improvements In Water Supply Systems Based On Optimization And Recognition Of Consumption Patterns. Journal of Engineering Science and Technology, volume 10, n. 5, pages 571-590, 2015.

G. Anjana, M. Kumar and B. Amrutur Comparison of Different Controllers for Equitable Water Supply in Water Networks. Computing and Control for the Water Industry, 2017.

J. WU and M. LU Research of secondary constant pressure water supply system based on fuzzy-PID controller. IEEE 3rd Information Technology and Mechatronics Engineering Conference, pages 462-465, 2017.

T. J. Ross Fuzzy logic with engineering applications. New York: Wiley, volume 2, 2004.

H. Alegre, W. Hirner, J. M. Baptista and R. Parena Indicadores de desempenho para serviços de abastecimento de água. Série Guias Técnicos, LNEC and IRAR, 2004.

R. O. S. Signoreti, R. Z. Camargo, L. M. Canno, M. S. G. Pires and L. C. L. J. Ribeiro Importance of pressure reducing valves (PRVs) in water supply networks. In Journal of Physics: Conference Series, volume 738, n. 1, page 012026, 2016, August. 